in vivo $35: 3563-3568(2021)$

doi:10.21873/invivo.12659

\title{
Impact of C-reactive Protein Flare Response in Patients With Advanced Urothelial Carcinoma Who Received Pembrolizumab
}

\author{
IKKO TOMISAKI $^{1 *}$, MIRII HARADA ${ }^{1 *}$, KEI TOKUTSU ${ }^{2}$, AKINORI MINATO $^{1}$, YUJIRO NAGATA $^{1}$, \\ RIEKO KIMURO $^{1}$, MASAHIRO MATSUMOTO ${ }^{1}$ and NAOHIRO FUJIMOTO ${ }^{1}$ \\ ${ }^{1}$ Department of Urology, University of Occupational and Environmental Health, Kitakyushu, Japan; \\ ${ }^{2}$ Department of Public Health, University of Occupational and Environmental Health, Kitakyushu, Japan
}

\begin{abstract}
Background/Aim: To clarify the clinical significance of the temporary elevated $C$-reactive protein $(C R P)$ levels followed by a decrease below baseline (CRP flare response) after administration of pembrolizumab to patients with advanced urothelial carcinoma (UC). Patients and Methods: We retrospectively reviewed 31 patients with advanced UC who received pembrolizumab. Patients were categorized into 3 groups (flare-responder, responder, nonresponder) according to early CRP kinetics. Intergroup tumor response and survivals were compared. Results: Objective response rates of flare-responder, responder, and non-responder groups were 75\%, 80\%, and 26\%, respectively. Median overall survival was not reached in flare-responder and responder groups, and was 10.2 months in the non-responder group ( $p=0.03)$. Furthermore, the flareresponder group did not reach median progression-free survival, and for the responder and non-responder groups it was 15.2 and 2.8 months, respectively $(p=0.03)$. Conclusion: CRP flare response might be a promising biomarker in patients with advanced UC who received pembrolizumab.
\end{abstract}

Platinum-based chemotherapy regimens are the gold standard treatment for patients with advanced urothelial carcinoma

This article is freely accessible online.

*These Authors contributed equally to this work.

Correspondence to: Ikko Tomisaki, Department of Urology, University of Occupational and Environmental Health, 1-1 Iseigaoka, Yahatanishi-ku, Kitakyushu City, Fukuoka, 807-8555, Japan. Tel: +81 936917446, Fax: +81 936038724, e-mail: ikko@med.uoeh-u.ac.jp

Key Words: Urothelial carcinoma, C-reactive protein (CRP), CRP flare response, pembrolizumab, immune checkpoint inhibitors, tumor microenvironment.
(UC). However, no standard second-line chemotherapy agents exist. In the KEYNOTE-045 phase 3 clinical trial, the survival benefit of pembrolizumab was investigated in patients with advanced UC that had recurred or progressed after platinum-based chemotherapy (1). According to recent guidelines for bladder cancer or upper urinary tract cancer, pembrolizumab is recommended as second-line therapy for patients who previously received platinum-based combination chemotherapy. Extended follow-up (median 27.7 months) data on pembrolizumab reported a median overall survival (OS) and objective response rate (ORR) of 10.1 months and $21.1 \%$, respectively (2), and the oncological benefits were limited. Additionally, some patients reported immune-related adverse events (irAEs); therefore, it is valuable to identify biomarkers in predicting the effectiveness of pembrolizumab.

Several predictive biomarkers have been identified in patients with advanced UC on pembrolizumab (3-8). Markers related to systemic inflammatory factors, including C-reactive protein (CRP) (3, 4, 6-8) or the neutrophile/lymphocyte ratio (5-7), were reported as useful. Previous studies showed CRP baseline levels $(3,7,8)$ or kinetics $(4,6)$ were associated with clinical outcomes. Furthermore, temporary elevation of CRP levels followed by a decrease below baseline (CRP flare response) was also investigated as one of the biomarkers in patients with metastatic renal cell carcinoma (RCC) (9). However, there are no data on the CRP flare response in patients with UC who are treated with immune-check point inhibitors (ICIs).

The aim of this study was to clarify the clinical significance of the CRP flare response in patients who received pembrolizumab for advanced UC.

\section{Patients and Methods}

Medical records of consecutive patients admitted to the University of Occupational and Environmental Health hospital with metastatic 
Table I. Patient characteristics.

\begin{tabular}{|c|c|c|c|c|c|c|}
\hline & & Total cohort & Flare-responder & Responder & Non-responder & $p$-Value \\
\hline \multirow{2}{*}{ Gender } & Male (\%) & $24(77)$ & $4(100)$ & $6(60)$ & $14(82)$ & 0.34 \\
\hline & Female $(\%)$ & $7(23)$ & $0(0)$ & $4(40)$ & $3(18)$ & \\
\hline Age & years old, median (IQR) & $71.0(67.0-78.5)$ & $72.0(66.8-78.5)$ & $77.0(68.3-81.0)$ & $71.0(67.0-77.0)$ & 0.57 \\
\hline \multirow[t]{2}{*}{ Smoking history } & Never $(\%)$ & $14(45)$ & $1(25)$ & $4(40)$ & $9(53)$ & 0.68 \\
\hline & Yes $(\%)$ & $17(55)$ & $3(75)$ & $6(60)$ & $8(47)$ & \\
\hline \multirow[t]{2}{*}{ ECOG-PS } & $0(\%)$ & $24(87)$ & $2(50)$ & $10(100)$ & $12(71)$ & 0.08 \\
\hline & $\geq 1(\%)$ & $7(23)$ & $2(50)$ & $0(0)$ & $5(29)$ & \\
\hline \multirow[t]{2}{*}{ Primary site } & Upper urinary tract $(\%)$ & $14(45)$ & $2(50)$ & $6(60)$ & $6(35)$ & 0.42 \\
\hline & Bladder (\%) & $17(55)$ & $2(50)$ & $4(40)$ & $11(65)$ & \\
\hline \multirow{2}{*}{$\begin{array}{l}\text { Resection of } \\
\text { primary site }\end{array}$} & No $(\%)$ & $15(48)$ & $2(50)$ & $6(60)$ & $7(54)$ & 0.78 \\
\hline & Yes $(\%)$ & $16(52)$ & $2(50)$ & $4(40)$ & $10(41)$ & \\
\hline \multirow[t]{2}{*}{ Prior chemotherapy } & Perioperative (\%) & $6(19)$ & $0(0)$ & $3(30)$ & $3(18)$ & 0.56 \\
\hline & Curative (\%) & $25(81)$ & $4(100)$ & $7(70)$ & $14(82)$ & \\
\hline \multirow{2}{*}{$\begin{array}{l}\text { Time from prior } \\
\text { chemotherapy }\end{array}$} & $<3$ months $(\%)$ & $14(45)$ & $2(50)$ & $3(30)$ & $9(53)$ & 0.60 \\
\hline & $\geq 3$ months $(\%)$ & $17(55)$ & $2(50)$ & $7(70)$ & $8(47)$ & \\
\hline \multirow[t]{2}{*}{ Liver metastasis } & Absence $(\%)$ & $23(74)$ & $4(100)$ & $8(80)$ & $11(65)$ & 0.47 \\
\hline & Presence $(\%)$ & $8(26)$ & $0(0)$ & $2(20)$ & $6(35)$ & \\
\hline \multirow[t]{2}{*}{ Hemoglobin } & $<10 \mathrm{~g} / \mathrm{dl}(\%)$ & $11(35)$ & $1(25)$ & $4(40)$ & $6(35)$ & 1.00 \\
\hline & $\geq 10 \mathrm{~g} / \mathrm{dl}(\%)$ & $20(65)$ & $3(75)$ & $6(60)$ & $11(65)$ & \\
\hline
\end{tabular}

IQR: Interquartile range; ECOG-PS: Eastern Cooperative Oncology Group performance status.

UC who received pembrolizumab as second-line or later therapy after platinum-based chemotherapy were retrospectively reviewed. Data were collected between March 2018 and March 2021. This study was approved by the Ethics Committee of the University of Occupational and Environmental Health, Japan (UOEHCRB21-048).

Treatment and follow-up examinations. Pembrolizumab (200 $\mathrm{mg} /$ body) was administered every 3 weeks and continued until disease progression or unacceptable adverse events. Blood tests, including CRP levels, were performed at baseline and at least every 3 weeks, on the same schedule as pembrolizumab administration. Chest and abdominal computed tomography (CT) scans were performed at baseline and after every 3 or 4 cycles to evaluate the therapeutic effect. Tumor response was defined according to the Response Evaluation Criteria in Solid Tumor (RECIST) version 1.1.

Categorization by early CRP kinetics. According to the previous report (9), patients were categorized into 3 groups as follows: flareresponder-CRP levels had more than doubled from baseline within 1 month after initiation of pembrolizumab (CRP flare) and then decreased below baseline within 3 months; responder-CRP levels decreased $\geq 30 \%$ from baseline within 3 months without CRP flare; non- responder-the remaining patients.

Statistical analysis. Patient characteristics were compared among the groups using Kruskal-Wallis or Fisher's exact tests for continuous or nominal variables, respectively. Tumor responses among the groups were compared using the Fisher's exact test. OS and progression-free survival (PFS) were calculated from the first date of pembrolizumab therapy to the date of the last follow-up or death from any cause, and disease progression, respectively. Survival curves were estimated using the Kaplan-Meier method and compared using the log-rank test. A $p$ value $<0.05$ was considered statistically significant. All statistical analyses were performed using
EZR (Saitama Medical Center, Jichi Medical University, Saitama, Japan), which is a graphical user interface for R (The R Foundation for Statistical Computing, Vienna, Austria) (10).

\section{Results}

A total of 39 patients with advanced UC received pembrolizumab as second-line or later therapy during the study period. One patient who had acute pyelonephritis 5 days after first administration of pembrolizumab and 7 patients who died within 3 months after starting pembrolizumab therapy were excluded. Patient characteristics, categorized by early CRP kinetics, are summarized in Table I. Of 31 eligible patients, four, 10, and 17 patients were divided into flareresponder, responder, and non-responder groups, respectively. No significant difference was observed in patient characteristics among the three groups.

Two of 17 patients in the non-responder group did not undergo CT scans because of their clinical progression, which included fatigue and anorexia. The best responses in each group are demonstrated in Figure 1. The ORR of the total cohort was $52 \%$, and those of the flare-responder, responder, and non-responder groups were $75 \%, 80 \%$, and $27 \%$, respectively. Progressive disease was not observed in the flare-responder group during the follow-up period. There was a significant difference in tumor responses among the three groups.

Median follow-up was 10.2 months [interquartile range (IQR) 5.1-15.0 months]. Progression was observed in 19 patients; of these, four patients received subsequent therapy 


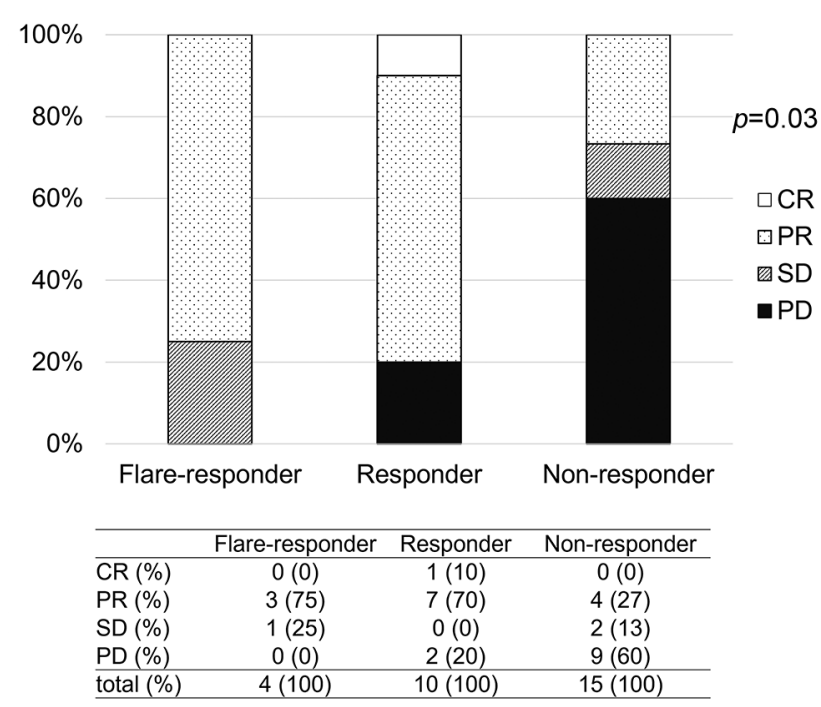

Figure 1. The best responses of each group categorized by early $C$ reactive protein kinetics. PD: Progressive disease; SD: stable disease; $C R$ : complete response; PR: partial response.

(gemcitabine-carboplatin: 3, paclitaxel-gemcitabine: 1). Eleven patients died of UC, and one patient died of other causes during the follow-up period. In survival analyses of all patients, the median OS and PFS were 15.9 [95\% confidence interval (CI) 10.2- not estimable (NE)] and 7.0 (95\% CI=2.8NE) months, respectively (Figure 2). In the survival curves stratified by early CRP kinetics, median OS was not reached in flare-responder $(95 \% \mathrm{CI}=\mathrm{NE}-\mathrm{NE})$ and responder groups $(95 \% \mathrm{CI}=7.3-\mathrm{NE})$, and it was reached at 10.2 months (95\% CI $=4.0-15.9)$ in the non-responder group $(p=0.03)$. In addition, median PFS was not reached in the flare-responder group $(95 \% \mathrm{CI}=\mathrm{NE}-\mathrm{NE})$, and it was $15.2(95 \% \mathrm{CI}=2.1-\mathrm{NE})$ and $2.8(95 \% \mathrm{CI}=1.9-12.6)$ months in the responder and nonresponder groups, respectively ( $p=0.03$ ) (Figure 3).

\section{Discussion}

This is the first study to investigate the CRP flare response in patients with advanced UC who received pembrolizumab. We observed a CRP flare response in patients with advanced UC who received pembrolizumab therapy as well as in patients with RCC who were treated with nivolumab. Furthermore, favorable ORR, PFS, and OS were observed in patients who had a CRP flare response.

Recently, various biomarkers including blood cell count markers (11) or nutritional status-based markers such as prognostic nutritional index (12) were reported in pembrolizumab therapy for patients with advanced UC. Several studies on UC have reported that elevated CRP levels before surgery (13), chemotherapy $(14,15)$, or ICIs treatment $(3,7,8)$ were associated with poor prognosis.
Additionally, CRP kinetics variably predict oncological outcomes; therefore, its potential as a dynamic variable has also been reported in patients with UC (15), including those who received pembrolizumab $(4,6)$.Interestingly, recent studies reported that early elevation of CRP levels after administration of ICIs was related with a favorable tumor response in non-small cell lung cancer (16), and that CRP flare response was associated with significant tumor shrinkage and improved survival outcomes in patients with metastatic RCC who received nivolumab (9). Although a CRP flare response was observed in our patients with advanced UC who received pembrolizumab, the percent of patients with a CRP flare response (13\%) was lower compared to patients with RCC $(26 \%)$ in the previous report (9). This finding might be associated with the different responses to immunotherapy between UC and RCC.

In this study, though favorable ORR, OS, and PFS were observed in patients with a CRP flare response, it was not possible to reveal the differences in oncological outcomes between flare-responder and responder groups using multivariable analysis because of the small number of patients and absence of progression or death in patients with a CRP flare response during the study period. However, patient backgrounds including the factors associated with treatment efficacy $(3-8,17)$ among each group were not significantly different, and the CRP flare response might be an important factor to predict favorable oncological outcomes. In clinical practice, therefore, pembrolizumab should be continued in patients with a CRP flare response, when irAEs are manageable.

In the tumor microenvironment, CRP is a surrogate marker of IL-6, which carries out immune-suppressive functions, via induction of regulatory $\mathrm{T}$ cells or myeloidderived suppressor cells, to suppress the antigen-presenting function of dendritic cells and macrophages $(18,19)$. However, it has been suggested that IL-6 also promotes tumor immunity, such as promotion of infiltration of cytotoxic T cells into the tumor (20) or activation of effective M1-type macrophages (21). Therefore, in the mechanisms that occur during CRP flare response, temporary CPR elevation might reflect the activation of tumor immunity, and the decrease in CRP levels below baseline might represent an improved immune tumor microenvironment. In the future, uncovering the detailed mechanisms in the CRP flare response would facilitate precision medicine development in ICIs treatment for UC.

There are several limitations in this study. First, the study population was small, and the follow-up period was limited. The ORR in our patients taking pembrolizumab was $52 \%$ compared to $21.1 \%$ in the KEYNOTE-045 study (1). In addition, the ORR of Japanese patients in the KEYNOTE045 study was $20 \%$ (22); therefore, some biases might be included in the results because of the retrospective nature of 

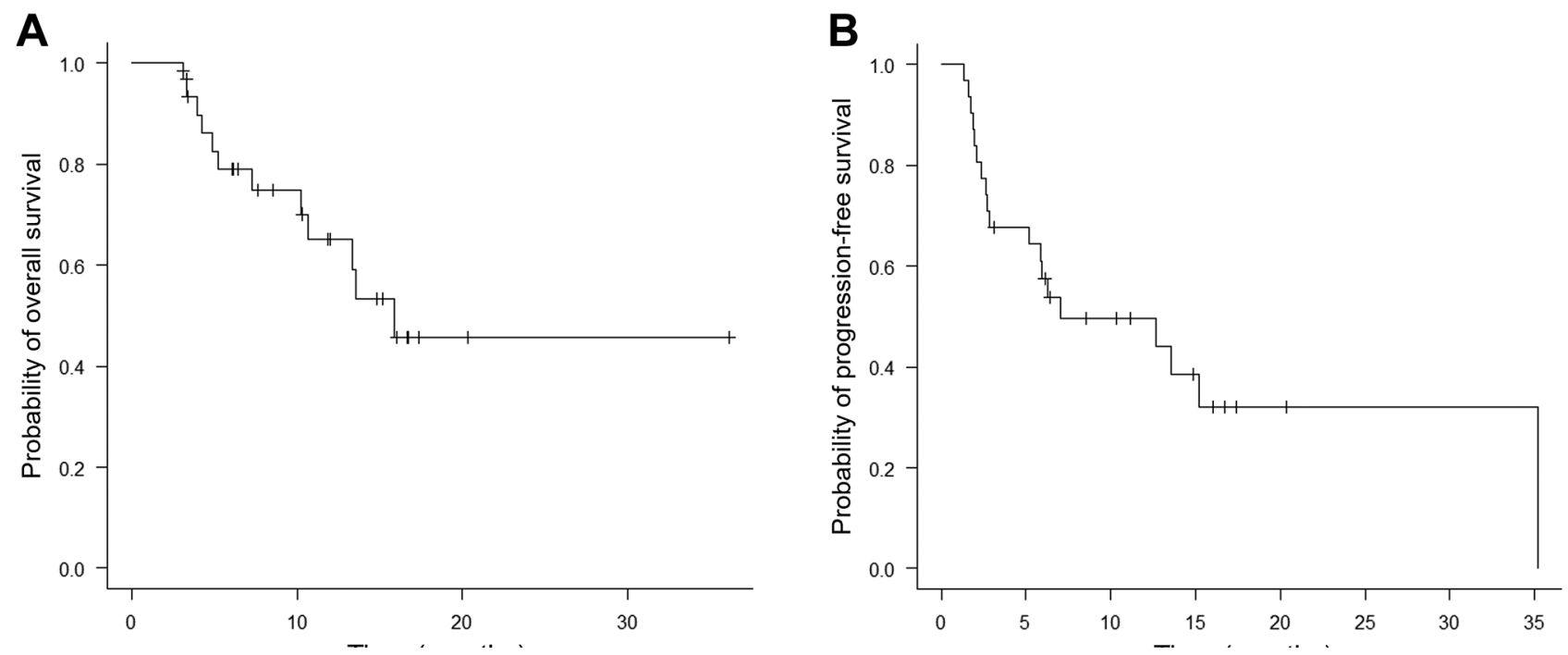

Figure 2. Kaplan-Meier estimates of A) overall survival and B) progression-free survival in the total cohort.

A

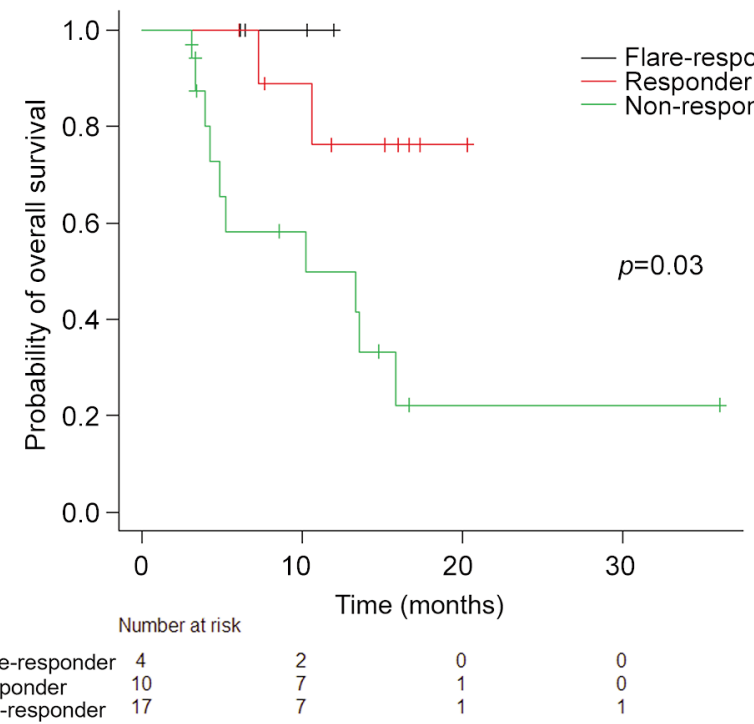

B

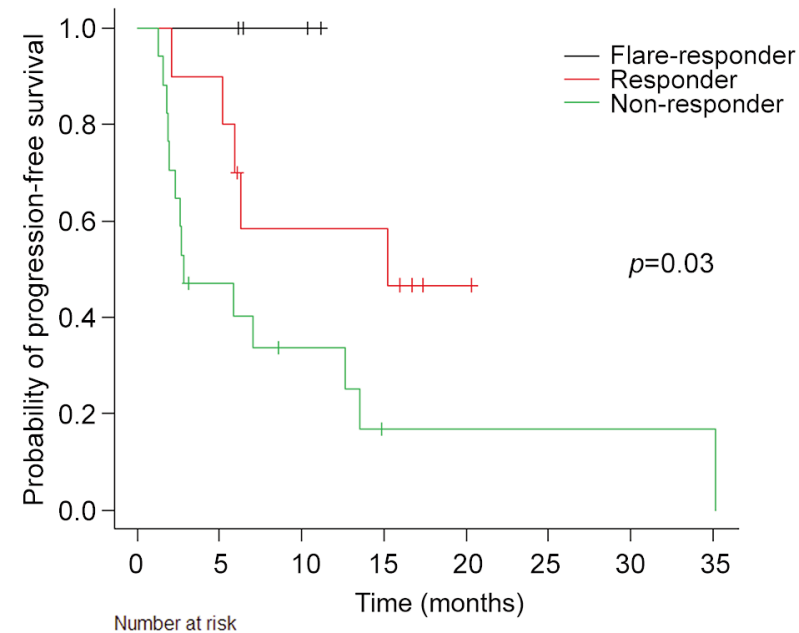

$\begin{array}{lcccccccc}\text { Flare-responder } & 4 & 4 & 2 & 0 & 0 & 0 & 0 & 0 \\ \text { Responder } & 10 & 9 & 5 & 5 & 1 & 0 & 0 & 0 \\ \text { Non-responder } & 17 & 7 & 4 & 1 & 1 & 1 & 1 & 1\end{array}$

Figure 3. Kaplan-Meier estimates of A) overall survival and B) progression-free survival in patients stratified by early C-reactive protein kinetics.

the study. Second, the patients were divided into three groups, according to CRP kinetics, following the previous study (9), and it is unclear whether this classification was adequate. Further study with more patients is necessary to reveal the optimal classification using CRP kinetics for patients with UC undergoing pembrolizumab therapy.

In conclusion, the CRP flare response was observed in $13 \%$ of patients with advanced UC who received pembrolizumab, and favorable oncological outcomes were observed in these patients. The CRP flare response might be a promising predictive and prognostic marker in patients with advanced UC who are on pembrolizumab therapy.

\section{Conflicts of Interest}

The Authors have no conflicts of interest in relation to this study.

\section{Authors' Contributions}

Conceptualization: Ikko Tomisaki, Mirii Harada; Methodology: Ikko Tomisaki, Mirii Harada, Kei Tokutsu; Formal analysis and 
investigation: Ikko Tomisaki, Mirii Harada, Kei Tokutsu, Akinori Minato, Yujiro Nagata, Rieko Kimuro, Masahiro Matsumoto; Writing - original draft preparation: Ikko Tomisaki, Mirii Harada; Writing - review and editing: Kei Tokutsu, Naohiro Fujimoto; Resources: Ikko Tomisaki, Mirii Harada; Supervision: Naohiro Fujimoto. All Authors read and approved the final manuscript.

\section{References}

1 Bellmunt J, de Wit R, Vaughn DJ, Fradet Y, Lee JL, Fong L, Vogelzang NJ, Climent MA, Petrylak DP, Choueiri TK, Necchi A, Gerritsen W, Gurney H, Quinn DI, Culine S, Sternberg CN, Mai Y, Poehlein CH, Perini RF, Bajorin DF and KEYNOTE-045 Investigators: Pembrolizumab as second-line therapy for advanced urothelial carcinoma. N Engl J Med 376(11): 10151026, 2017. PMID: 28212060. DOI: 10.1056/NEJMoa1613683

2 Fradet Y, Bellmunt J, Vaughn DJ, Lee JL, Fong L, Vogelzang NJ, Climent MA, Petrylak DP, Choueiri TK, Necchi A, Gerritsen W, Gurney H, Quinn DI, Culine S, Sternberg CN, Nam K, Frenkl TL, Perini RF, de Wit R and Bajorin DF: Randomized phase III KEYNOTE-045 trial of pembrolizumab versus paclitaxel, docetaxel, or vinflunine in recurrent advanced urothelial cancer: results of $>2$ years of follow-up. Ann Oncol 30(6): 970-976, 2019. PMID: 31050707. DOI: 10.1093/annonc/ mdz127

3 Fujiwara M, Yuasa T, Urasaki T, Komai Y, Fujiwara R, Numao $\mathrm{N}$, Yamamoto $\mathrm{S}$ and Yonese J: Effectiveness and safety profile of pembrolizumab for metastatic urothelial cancer: A retrospective single-center analysis in Japan. Cancer Rep (Hoboken): e1398, 2021. PMID: 33934570. DOI: $10.1002 / \mathrm{cnr} 2.1398$

4 Kijima T, Yamamoto H, Saito K, Kusuhara S, Yoshida S, Yokoyama M, Matsuoka Y, Numao N, Sakai Y, Matsubara N, Yuasa T, Masuda H, Yonese J, Kageyama Y and Fujii Y: Early C-reactive protein kinetics predict survival of patients with advanced urothelial cancer treated with pembrolizumab. Cancer Immunol Immunother 70(3): 657-665, 2021. PMID: 32876736. DOI: $10.1007 / \mathrm{s} 00262-020-02709-2$

5 Kobayashi T, Ito K, Kojima T, Kato M, Kanda S, Hatakeyama S, Matsui Y, Matsushita Y, Naito S, Shiga M, Miyake M, Muro Y, Nakanishi S, Kato Y, Shibuya T, Hayashi T, Yasumoto H, Yoshida T, Uemura M, Taoka R, Kamiyama M, Ogawa O, Kitamura H, Nishiyama $\mathrm{H}$ and Japan Urological Oncology Group: Risk stratification for the prognosis of patients with chemoresistant urothelial cancer treated with pembrolizumab. Cancer Sci 112(2): 760-773, 2021. PMID: 33283385. DOI: $10.1111 /$ cas. 14762

6 Tamura D, Jinnouchi N, Abe M, Ikarashi D, Matsuura T, Kato R, Maekawa S, Kato Y, Kanehira M, Takata R and Obara W: Prognostic outcomes and safety in patients treated with pembrolizumab for advanced urothelial carcinoma: experience in real-world clinical practice. Int J Clin Oncol 25(5): 899-905, 2020. PMID: 31907720. DOI: 10.1007/s10147-019-01613-9

7 Yamamoto Y, Yatsuda J, Shimokawa M, Fuji N, Aoki A, Sakano S, Yamamoto M, Suga A, Tei Y, Yoshihiro S, Kitahara S, Nagao K, Takai K, Kamiryo Y, Akao J, Yamaguchi S, Oba K, Shimabukuro T, Matsumoto H, Kamba T and Matsuyama H: Prognostic value of pre-treatment risk stratification and posttreatment neutrophil/lymphocyte ratio change for pembrolizumab in patients with advanced urothelial carcinoma.
Int J Clin Oncol 26(1): 169-177, 2021. PMID: 32948925. DOI: 10.1007/s10147-020-01784-w

8 Yasuoka S, Yuasa T, Nishimura N, Ogawa M, Komai Y, Numao $\mathrm{N}$, Yamamoto S, Kondo Y and Yonese J: Initial experience of pembrolizumab therapy in japanese patients with metastatic urothelial cancer. Anticancer Res 39(7): 3887-3892, 2019. PMID: 31262917. DOI: 10.21873/anticanres.13539

9 Fukuda S, Saito K, Yasuda Y, Kijima T, Yoshida S, Yokoyama M, Ishioka J, Matsuoka Y, Kageyama Y and Fujii Y: Impact of C-reactive protein flare-response on oncological outcomes in patients with metastatic renal cell carcinoma treated with nivolumab. J Immunother Cancer 9(2): e001564, 2021. PMID: 33602695. DOI: $10.1136 /$ jitc-2020-001564

10 Kanda Y: Investigation of the freely available easy-to-use software 'EZR' for medical statistics. Bone Marrow Transplant 48(3): 452-458, 2013. PMID: 23208313. DOI: 10.1038/bmt. 2012.244

11 Kadono Y, Kawaguchi S, Nohara T, Shigehara K, Izumi K, Kamijima T, Seto C, Takano A, Yotsuyanagi S, Nakagawa R, Miyagi T, Aoyama S, Asahi H, Fukuda R and Mizokami A: Blood cell count biomarkers predicting efficacy of pembrolizumab as second-line therapy for advanced urothelial carcinoma. Anticancer Res 41(3): 1599-1606, 2021. PMID: 33788755. DOI: 10.21873 /anticanres.14921

12 Ishiyama Y, Kondo T, Nemoto Y, Kobari Y, Ishihara H, Tachibana H, Yoshida K, Hashimoto Y, Takagi T, lizuka J and Tanabe K: Predictive impact of prognostic nutritional index on pembrolizumab for metastatic urothelial carcinoma resistant to platinum-based chemotherapy. Anticancer Res 41(3): 1607-1614, 2021. PMID: 33788756. DOI: 10.21873/anticanres.14922

13 Tanaka N, Kikuchi E, Shirotake S, Kanao K, Matsumoto K, Kobayashi H, Miyazaki Y, Ide H, Obata J, Hoshino K, Hayakawa N, Ito Y, Kosaka T, Kodaira K, Oyama M, Miyajima A, Momma T, Nakagawa K, Ueno M and Oya M: The predictive value of $\mathrm{C}$-reactive protein for prognosis in patients with upper tract urothelial carcinoma treated with radical nephroureterectomy: a multi-institutional study. Eur Urol 65(1): 227-234, 2014. PMID: 23219372. DOI: 10.1016/j.eururo. 2012.11.050

14 Eggers H, Seidel C, Schrader AJ, Lehmann R, Wegener G, Kuczyk MA and Steffens S: Serum C-reactive protein: a prognostic factor in metastatic urothelial cancer of the bladder. Med Oncol 30(4): 705, 2013. PMID: 24005810. DOI: 10.1007/ s12032-013-0705-6

15 Saito K, Urakami S, Komai Y, Yasuda Y, Kubo Y, Kitsukawa S, Okubo Y, Yamamoto S, Yonese J and Fukui I: Impact of Creactive protein kinetics on survival of patients with advanced urothelial carcinoma treated by second-line chemotherapy with gemcitabine, etoposide and cisplatin. BJU Int 110(10): 14781484, 2012. PMID: 22520732. DOI: 10.1111/j.1464-410X. 2012.11153.x

16 Ozawa Y, Amano Y, Kanata K, Hasegwa H, Matsui T, Kakutani T, Koyauchi T, Tanahashi M, Niwa H, Yokomura K and Suda T: Impact of early inflammatory cytokine elevation after commencement of PD-1 inhibitors to predict efficacy in patients with non-small cell lung cancer. Med Oncol 36(4): 33, 2019. PMID: 30825015. DOI: 10.1007/s12032-019-1255-3

17 Sonpavde G, Pond GR, Fougeray R, Choueiri TK, Qu AQ, Vaughn DJ, Niegisch G, Albers P, James ND, Wong YN, Ko YJ, Sridhar SS, Galsky MD, Petrylak DP, Vaishampayan UN, Khan 
A, Vogelzang NJ, Beer TM, Stadler WM, O'Donnell PH, Sternberg CN, Rosenberg JE and Bellmunt J: Time from prior chemotherapy enhances prognostic risk grouping in the secondline setting of advanced urothelial carcinoma: a retrospective analysis of pooled, prospective phase 2 trials. Eur Urol 63(4): 717-723, 2013. PMID: 23206856. DOI: 10.1016/j.eururo. 2012.11.042

18 Hunter CA and Jones SA: IL-6 as a keystone cytokine in health and disease. Nat Immunol 16(5): 448-457, 2015. PMID: 25898198. DOI: $10.1038 /$ ni.3153

19 Tsukamoto H, Fujieda K, Senju S, Ikeda T, Oshiumi H and Nishimura Y: Immune-suppressive effects of interleukin-6 on Tcell-mediated anti-tumor immunity. Cancer Sci 109(3): 523-530, 2018. PMID: 29090850. DOI: 10.1111/cas.13433

20 Fisher DT, Chen Q, Skitzki JJ, Muhitch JB, Zhou L, Appenheimer MM, Vardam TD, Weis EL, Passanese J, Wang WC, Gollnick SO, Dewhirst MW, Rose-John S, Repasky EA, Baumann $\mathrm{H}$ and Evans SS: IL-6 trans-signaling licenses mouse and human tumor microvascular gateways for trafficking of cytotoxic T cells. J Clin Invest 121(10): 3846-3859, 2011. PMID: 21926464. DOI: 10.1172/JCI44952
21 Beyranvand Nejad E, Labrie C, van Elsas MJ, Kleinovink JW, Mittrücker HW, Franken KLMC, Heink S, Korn T, Arens R, van Hall T and van der Burg SH: IL-6 signaling in macrophages is required for immunotherapy-driven regression of tumors. J Immunother Cancer 9(4): e002460, 2021. PMID: 33879600. DOI: $10.1136 /$ jitc-2021-002460

22 Nishiyama H, Yamamoto Y, Sassa N, Nishimura K, Fujimoto K, Fukasawa S, Yokoyama M, Enokida H, Takahashi K, Tanaka Y, Imai K, Shimamoto T, Perini R, Frenkl T, Bajorin D and Bellmunt J: Pembrolizumab versus chemotherapy in recurrent, advanced urothelial cancer in Japanese patients: a subgroup analysis of the phase 3 KEYNOTE-045 trial. Int J Clin Oncol 25(1): 165-174, 2020. PMID: 31729625. DOI: 10.1007/s10147019-01545-4

Received August 13, 2021

Revised September 11, 2021

Accepted September 13, 2021 